\section{PWE-083 ARE FUNCTIONAL CONSTIPATION AND CONSTIPATION-SUBTYPE IRRITABLE BOWEL SYNDROME DISTINCT WITH RESPECT TO 5-HYDROXYTRYPTAMINE SIGNALLING AND MOTOR- SENSORY FUNCTION?}

doi:10.1136/gut.2011.239301.346

C Shekhar, ${ }_{1}^{1, *}$ P Monaghan, ${ }^{2}$ J Adaway, ${ }^{2}$ J Morris, ${ }^{3}$ P Whorwell, ${ }^{1}$ B Keevil, ${ }^{2}$ L A Houghton ${ }^{1,4}{ }^{1}$ Neurogastroenterology Unit, University of Manchester, Manchester, UK; ${ }^{2}$ Biochemistry Department, UHSM, Manchester, UK; ${ }^{3}$ Statistics, UHSM, Manchester, UK; ${ }^{4}$ College of Medicine, Mayo Clinic, Florida, USA

Introduction Recent studies suggest that patients identified by Rome III criteria for functional constipation (FC) and irritable bowel syndrome with constipation (IBS-C) are not distinct groups. We have shown that patients with IBS-C exhibit limited 5-HT response to meal ingestion, with plasma concentrations remaining similar to fasting. Our aim was to determine whether patients with FC show a similar 5-HT meal response as IBS-C, and to investigate relationships to motor-sensory function.

Methods 23 IBS-C patients (aged 19-50 years; Rome III), 11 FC patients (25-46 years; Rome III) and 23 healthy volunteers (HV) (20-49 years) were recruited. Plasma 5-HT concentrations were measured under fasting $(2 \mathrm{~h})$ and fed $(4 \mathrm{~h})$ conditions. Within 2 weeks, oro-caecal (hydrogen breath) and colonic (radio-opaque markers followed by X-ray) transit, along with rectal sensitivity (barostat) were determined.

Results FC and IBS-C had similarly reduced 5-HT responses to meal ingestion (mean increase from fasting (SD), FC: -1.1 $\mathrm{nmol} / 1$ ( \pm 6.9$), \mathrm{p}=0.1$; IBS-C: $-1.9 \mathrm{nmol} / 1$ ( \pm 7.0$), \mathrm{p}=0.02) \mathrm{com}-$ pared with $\mathrm{HV}(+4.7 \mathrm{nmol} / \mathrm{l}( \pm 9.6))$, but comparable fasting 5-HT concentrations (FC: $34.2 \mathrm{nmol} / 1$ ( \pm 13.2$)$; IBS-C: 27.8 nmol/1 ( \pm 17.3$)$; HV: 27.0 nmol/1 ( \pm 9.6$)$ ). Likewise, FC and IBS-C have reduced colonic (FC: $61.6 \mathrm{~h}( \pm 17.9), \mathrm{p}=0.001$; IBS-C: 55.6 h $( \pm 18.5), p=0.001$ vs HV: 34.6 h $( \pm 17.8))$ but not orocecal ((FC: $321.4 \mathrm{~min}( \pm 96.6)$; IBS-C: $311.7 \mathrm{~min}( \pm 96.6)$ vs HV: $301.8 \mathrm{~min}$ $( \pm 87.5))$ transit compared with HV. Only rectal sensitivity differed, with IBS-C exhibiting lower pain thresholds (23.4 $\mathrm{mm} \mathrm{Hg}( \pm 8.3), \mathrm{p}=0.03)$ but not FC $(32.7 \mathrm{~mm} \mathrm{Hg}( \pm 12.2)$ compared with $\mathrm{HV}(30.7 \mathrm{~mm} \mathrm{Hg}( \pm 8.2))$. Moreover, although the 5 -HT meal response was similar between hyper- $(-0.7 \mathrm{nmol} / 1$ $( \pm 4.9))$, normo- $(-1.5 \mathrm{nmol} / \mathrm{l}( \pm 7.5)$ and hypo- $(-4.7 \mathrm{nmol} / 1$ $( \pm 7.8))$ sensitive constipated patients, those with hypo-sensitivity (FC (27\%) and IBS-C (4\%)) had higher fasting and fed 5 -HT concentrations (fasting: $44.3 \mathrm{nmol} / 1$ ( \pm 17.2$)$, fed: 39.6 $\mathrm{nmol} / 1$ ( \pm 20.8$), \mathrm{p}=0.001$ and $\mathrm{p}=0.08$, respectively) compared with HV (24.7 nmol/1 ( \pm 7.5$), 27.9 \mathrm{nmol} / \mathrm{l}( \pm 9.5))$. Hyper- $(20.4$ $\mathrm{nmol} / \mathrm{l}( \pm 7.6), 19.7 \mathrm{nmol} / \mathrm{l}( \pm 5.6))$ and normo- $(30.7 \mathrm{nmol} / \mathrm{l}$ $( \pm 16.6), 29.2 \mathrm{nmol} / \mathrm{l}( \pm 16.8))$ sensitive patients were no different from HV.

Conclusion There appears to be no distinction between FC and IBS-C patients with respect to 5-HT meal response and GI transit, although IBS-C patients are more viscerally sensitivity. Hypo-sensitive constipated patients appear to have a distinct 5-HT profile.

Competing interests None.

Keywords 5 HT, Constipation, Functional Gastrointestinal Disorders (FGID), IBS, Rectal Sensitivity, ROME III. 


\section{BSG abstracts}

\section{REFERENCES}

1. Am J Gastroenterol 2010;105:2228-34.

2. Gastroenterology 2006;130:34-43

3. J Chromatogr B 2009;877:2163 